\title{
Study on Parameters Optimization Method of Fuzzy Neural Network PID Controller
}

\author{
Chunguang Zhang and Lifang Zhang \\ School of Electronics and Information Engineering, Dalian Jiaotong \\ University,Dalian 116028 China \\ zhangcglf@163.com
}

\begin{abstract}
In allusion to the insufficient of the traditional parameters optimization of the traditional fuzzy neural network PID controller, the parallel search characteristics of the ant colony algorithm in the whole parameter space is used. A parameters optimization method of the PID controller based combining the ant colony algorithm and fuzzy theory and neural network is proposed in this paper. The method used the ant colony algorithm to comprehensively optimize the parameters and structure of fuzzy neural network, which to be used to train and determine the parameters of the PID controller in order to get the fuzzy neural network PID controller. This method is used in the practical application of nonlinear coupled system, the experimental results show that the optimized fuzzy neural network PID controller takes the faster approximation control objectives, the shorter response time, the smaller overshoot and higher control accuracy. Consequently, the research has the theoretical significance and practical application value.
\end{abstract}

Keywords: Artificial intelligence technology, Fuzzy neural network, Parameter optimization, PID controller, Performance analysis

\section{Introduction}

The conventional proportional integral differential (PID) control is one of the most widely control strategy in the industrial control application [1]. The PID takes on the simple control structure, easy adjusting the parameters on line, good robustness and high reliability and so on. It is widely applied in linear control system, especially is suitable for the deterministic control system of establishing precise mathematical model [2-4]. However, the mechanism of the controlled object in the real industrial environment is complex, and it takes on many shortcomings, such as the nonlinear, time-varying uncertainty, pure lag and so on. Under the influence of the actual noise and load disturbance and so on, the conventional PID controller is often bad tuning, poor performance. And the tuned parameters can't be adjusted on line; the adaptation ability is poor for operating condition.

With the appearance, development and application of the intelligent control theory and artificial intelligence techniques [5], such as fuzzy theory, rough set theory, neural networks and genetic algorithms, particle swarm optimization, ant colony optimization algorithm, simulated annealing algorithm and so on, these methods has opened up new avenues to control the PID control of the complex system[6]. Li et al. proposed a new type of multivariable fuzzy control algorithm based on fuzzy control theory in order to effectively solve the control problem of the ball mill pulverizing system [7]. Zhao achieved a decoupling 
algorithm based combining the decoupling theory and neural network [8]. And a new mill control system was designed according to this algorithm. With the intelligent control theory, artificial intelligence techniques such as fuzzy theory, rough set theory, neural network, etc., and a new optimization algorithm such as genetic algorithms, particle swarm optimization, ant colony optimization algorithm, simulated annealing algorithm emergence, development and application of control PID control of complex systems has opened up new avenues. However, due to these defects of the slower learning rate and poor dynamic performance of the neural network, the neural network can't effectively eliminate static coupling in the controlled object. So it is not conducive to realize on the engineering. Fu et al proposed a new model of the neuron network, named PID neuron network (PIDNN) [9]. The neuron of this model in the hidden layer takes on the input characteristics and output characteristics of the PID controller. It consists of the proportion (P), integral (I), differential (D) neurons. The PID controller combines the characteristics of neural network. For the multivariable time-varying coupling system, it adjusts the connection weights of system output by the online learning. When the object parameter changes, it will adjust the interaction among three neurons in order to make the decoupled system with good dynamic and static performance, be compatible to control the nonlinear multivariable system.

In order to improve the performance of the fuzzy neural network (FNN), the ACO algorithm is used to optimize the structure and parameters of the FNN in order to obtain the optimized FNN, which is used to control the parameters of the PID controller. An optimized fuzzy neural network PID controller is proposed in this paper. In this method, the FNN is used to realize the fuzzy reasoning of the original fuzzy controller and adjust membership function in order to obtain the stronger learning and adaptive capacity of the PID controller. The effectiveness of the controller and its algorithm are verified by using Matlab platform. The simulation results show that the proposed method has improved on accuracy, search capability, stability, robustness and so on and obtained good control effect.

\section{Artificial Intelligence Technology}

\subsection{Ant colony algorithm}

Ant colony algorithm (ACO) was introduced by Marco Dorigo[10]. The ACO is a metaheuristic inspired by the behaviors of real ants in their search for the shortest path to food. The ACO algorithm consists of a number of cycles (iterations) of solution construction. In iteration, a number of ants construct complete solutions by using heuristic information and the collected experiences of previous groups of ants. These collected experiences are represented by the pheromone trail which is deposited on the constituent elements of a solution. Small quantities are deposited during the construction phase while larger amounts are deposited at the end of iteration in proportion to solution quality. Pheromone can be deposited on the components and/or the connections used in one solution depending on the problem. The procedure of pheromone update rule is shown:

(1) The transition rule

In the route, the $\mathrm{k}^{\text {th }}$ ant starts from city $\mathrm{r}$, the next city $\mathrm{s}$ is selected among the unvisited cities memorized in $J_{r}^{k}$ according to the following formula:

$$
s=\underset{u \in J_{r}^{k}}{\arg \max }\left[\tau_{i}(r, u)^{\alpha} \cdot \eta(r, u)^{\beta}\right] \text { if } q \leq q_{0}(\text { Exploitation })
$$

To visit the next city $\mathrm{s}$ with the probability $p_{k}(r, s)$, 


$$
p_{k}(r, s)= \begin{cases}\frac{\tau(r, s)^{\alpha} \cdot \eta(r, s)^{\beta}}{\sum_{u \in J_{r}^{k}} \tau(r, u)^{\alpha} \cdot \eta(r, u)^{\beta}} & \text { if } s \in J_{r}^{k} \\ 0 & \text { otherwise if } q>q_{0} \text { (Bias Exploitation) }\end{cases}
$$

In two formula, $p_{k}(r, s)$ is the transition probability, $\tau(r, u)$ is the intensity of pheromone between city $\mathrm{r}$ and city $\mathrm{u}$ in the $\mathrm{i}^{\text {th }}$ group, $\eta(r, u)$ is the length of the path from city $\mathrm{r}$ to city $\mathrm{u}$, $J_{r}^{k}$ is the set of unvisited cities of the $\mathrm{k}^{\text {th }}$ ant in the $\mathrm{i}^{\text {th }}$ group, the parameter $\alpha$ and $\beta$ are the control parameters, $\mathrm{q}$ is a uniform probability $[0,1]$.

(2) The pheromone update rule

In order to improve the solution, the pheromone trails must be updated. Trail updating includes local updating and global updating. The local trail updating formula is given by:

$$
\tau(r, u)=(1-\rho) \tau(r, s)+\sum_{k=1}^{m} \Delta \tau_{k}(r, s)
$$

In the formula (3), $\rho(0<\rho<1)$ is the pheromone trial evaporating rate. $\Delta \tau_{k}(r, s)$ is the amount of pheromone trail added to the edge(r,s ) by ant $k$ between time $t$ and $\mathrm{t}+\Delta \mathrm{t}$ in the tour. It is given by:

$$
\Delta \tau_{k}(r, s)= \begin{cases}\frac{Q}{L_{k}} & (\mathrm{r}, s) \in \pi_{k} \\ 0 & \text { otherwise }\end{cases}
$$

where $Q$ is a constant parameter, $L_{k}$ is the distance of the sequence $\pi_{k}$ toured by ant in $\Delta t$.

\subsection{Fuzzy neural network}

Fuzzy theory and neural network model are found without the mathematics model of dynamic characteristic Fuzzy theory is to describe the regular expert knowledge, experience or operation data, and the neural network is used to train sample data. Fuzzy neural network is to combine the fuzzy theory and neural network. The FNN combines the advantages of fuzzy theory and neural network. It takes on great advantages for dealing with the non-linear and fuzziness and so on. In intelligent information processing, the FNN has a huge potential, more and more experts and scholars contribute to this area and the fruitful research results are obtained in the recent years. The FNN model is composed of five layers. The function of each layer is illuminated [11]:

(1) Input layer In this layer, input vectors may be accurate numerical vector or fuzzy value. The followed expression is:

$$
O_{i}^{1}=I_{i}=x_{i}, i=1,2,3, \cdots, n
$$

(2) Fuzzification layer In this layer, the function is to fuzzify the input variable. The membership function adopts Gaussian function because of providing with the good smoothness of the Gaussian function. The followed expression is: 


$$
\mu_{i j}=\exp \left[-\frac{\left(x_{i}-c_{i j}\right)^{2}}{\sigma_{i j}^{2}}\right], i=1,2, \cdots, r ; j=1,2, \cdots, u
$$

where in the $\mathrm{j}^{\text {th }}$ neuron, the $\mu_{i j}$ is the $\mathrm{i}^{\text {th }}$ Gaussian function, the $\sigma_{i j}$ is the $\mathrm{i}^{\text {th }}$ Gaussian function standard deviation, the $c_{i j}$ is the $\mathrm{i}^{\text {th }}$ Gaussian function center, $r$ is the number of input dimension, $u$ is the number of neuron. The output result of the $\mathrm{j}^{\text {th }}$ neuron is shown:

$$
O_{j}^{2}=\exp \left[\sum_{i=1}^{r} \frac{\left(x_{i}-c_{i j}\right)^{2}}{\sigma_{i j}^{2}}\right], j=1,2, \cdots, u
$$

(3) Fuzzy reasoning layer or fuzzy membership layer In this layer, one neuron denotes on fuzzy rule. The followed expression is:

$$
O_{j}^{3}=w_{a 1} O_{1}^{2} \times w_{a 2} O_{2}^{2} \times \cdots \times w_{a u} O_{u}^{2}=\prod_{j=1}^{u} w_{a j} O_{j}^{2}
$$

(4) Anti-fuzzification layer In this layer, the clear output of the fuzzy neural network is realized and the anti-fuzzification algorithm is provided with the global approximability. We adopt the general fuzzy method of the gravity solution in this paper. The expression is:

$$
O_{j}^{4}=\frac{O_{j}^{3}}{\sum_{j=1}^{u} O_{j}^{3}}, j=1,2, \cdots, u
$$

(5) Output layer The precision calculation is realized by the followed expression:

$$
y=\sum_{j=1}^{u} w_{b j} O_{j}^{4}, \quad j=1,2, \cdots, u
$$

where, $w_{b j}$ is the connection weight of the FNN model.

\section{The ACO Algorithm Optimizes the Fuzzy Neural Network}

\subsection{The idea of optimizing the fuzzy neural network}

The optimization of the fuzzy neural network mainly includes the structure optimization and parameter optimization of the FNN. The structure optimization of the FNN is to search the optimal network structure of the meeting accuracy requirement under the given divided numbers of the input and output space. The parameter optimization is to determine the optimal network parameters of the FNN by using the appropriate algorithm. The structure of the FNN typically includes the number of the neurons in each layer and the connection number between the adjacent neurons. For the fuzzy neural network, the neurons are changing in the only third layer. And whether one neuron plays a role, we may judge the neuron whether or not have the connection with the neuron of the adjacent layer. As a result, the structure optimization of the FNN is to determine the numbers of the nodes in the third layer, the connection numbers and the connection weights between the third layer and the second layer, and the third layer and the fourth layer. 


\subsection{The steps for optimizing the FNN}

In the optimized method of the fuzzy neural network, the parameters of the proposed method are initialized and search for the optimization solution by the search space. During processing, the ACO algorithm is used to execute an evolution of the solution. The optimized method of the fuzzy neural network by using the ACO algorithm is described:

Step1: initialization

Generate $\mathrm{g}$ groups of ants $\left(g_{0}, g_{1} \ldots\right.$ and $\left.g_{g}\right)$.Each group has $N$ ants and each ant chooses one city as its starting city. According to the following equation, the initial pheromone level among any two cities is set $T(i)$ :

$$
T(i)=k \bullet a^{-f\left(X_{i}\right)}
$$

where $\mathrm{k}$ is a constant $(k>0), 0<a<1, f\left(X_{i}\right)$ is the objective function value. The values of the ' $k$ ' and ' $a$ ' are determined according to the actual problem.

Step 2. Calculate transition probability

The $k^{\text {th }}$ ant of the $i^{\text {th }}$ group constructs its traveling sequence of cities by using the transition rule expression.

Step 3. Compute the length of the path for each ant.

Step 4. Update the amount of pheromone

The $k^{\text {th }}$ ant of the $i^{\text {th }}$ group allocates its traveling sequence of cities by using the local pheromone update rule expression according to the length of its path.

Step 5. Output optimization solution

Compute whether a better solution is obtained in this step than the last step; if so, then perform a global update on the solution and go into step 6. Otherwise repeat step 2 to step5.

Step 6. Optimize the structure of the FNN

The structure of the FNN includes the number of the nodes and the related connection weights of the nodes in the hidden layer. Suppose the given learning sample $\{x(i), \bar{y}(i), i=1,2,3, \cdots, N\}$, the error of one sampling $\{x, \bar{y}\}$ is defined as: $e=y-\bar{y}$. The objective function is defined as:

$$
J(\bullet)=\frac{1}{2} \sum_{i=1}^{N}[y(i)-\bar{y}(i)]^{2}=\frac{1}{2} \sum_{i=1}^{N} e^{2}(i)
$$

Step 7. Optimize the parameters of the FNN

The key of the parameters of the FNN is to find the learning parameters of the FNN. A unit step function $\delta$ is introduced into this paper. 


$$
\delta(\xi)= \begin{cases}0, & \xi>0.5 \\ 1, & \xi \leq 0.5\end{cases}
$$

where $\xi$ is he switch parameter, its value is $[0,1]$. The unit step function $\delta(\bullet)$ is to add a structural parameter $0 / 1$ into the learning parameter. The optimization problem of the FNN is converted to a pure parametric optimization problem. So the ACO algorithm is used to optimize the structure optimization and parameter optimization of the FNN.

\section{Step 8. Train the FNN}

Input the training samples to train the corresponding FNN of each individual. The optimization process of connection weights of the FNN is an iterative process. In the optimization process of connection weights of the FNN, the given sample set is classified in order to guarantee that the training adopts the training set, which is not the same.

Step 9. Calculate the error

Suppose the total number of training samples is $\mathrm{T}$, the mean value of the average error of the optimized FNN is:

$$
\varepsilon_{A V G}=\frac{1}{K} \sum_{j \in n} e_{j}^{2}(k)
$$

where $e_{i}(k)=d_{i}(k)-y_{i}(k), . e_{i}(k)$ is the error signal of the unit.

Step 10. Judge termination condition

When the objective function value (MSE) meets the accuracy requirement, the method will end. Otherwise go into Step 6 to continue to train the FNN until the maximum iteration.

\subsection{Simulation experiment for optimizing FNN}

In order to test the effectiveness of the optimized fuzzy neural network by using ACO algorithm, the simulation experiments are implemented in this paper. First, the initial value and the related parameters of the ACO algorithm are determined by some preliminary experiments in order to obtain the best performance for the ACO algorithm. According to the different size of the colony, the performance influence of the algorithm is tested. Because the number of calculation fitness remains the same, the selected size of the colony is larger, the corresponding number of iteration is less. So the parameters of the ACO algorithm are set: the initial ant size is 20 , the number of iteration is 1000 , the pheromone factor is 1 , the heuristic factor is 2 , the pheromone volatility coefficient is 0.05 , and the amount of pheromone is 80 . The experimental simulation results are shown in Fig.1 Fig.3.

From the Figure 1, we can see, when the iteration is 278 , the fitness value is becoming to be stable, the stable value is 24.78 . As can be seen from Figure 2, the prediction output is very close to the expected output by using the optimized FNN. And the prediction output is equal to the expected output in the individual iteration. As can be seen from Figure 3, the prediction error percentage of the FNN is extremely small. 


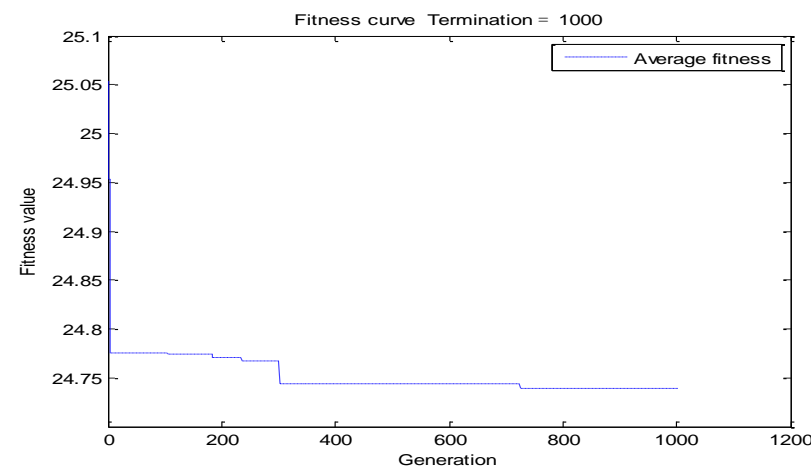

Figure 1. Fitness curve of the optimizing FNN using the ACO

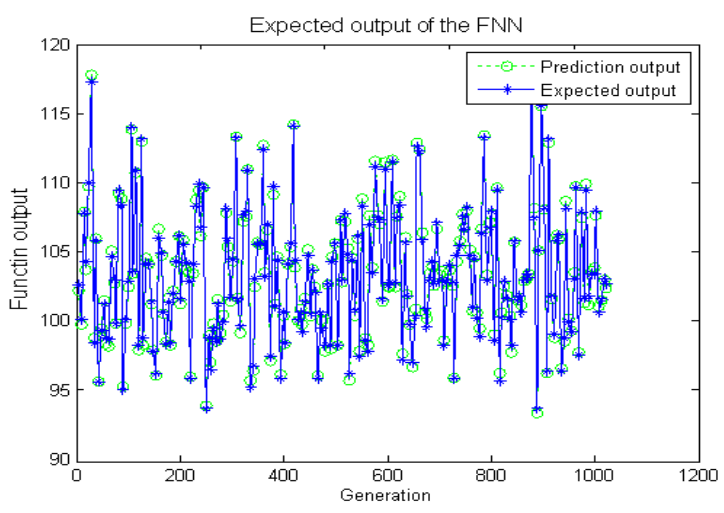

Figure 2. Comparison the output

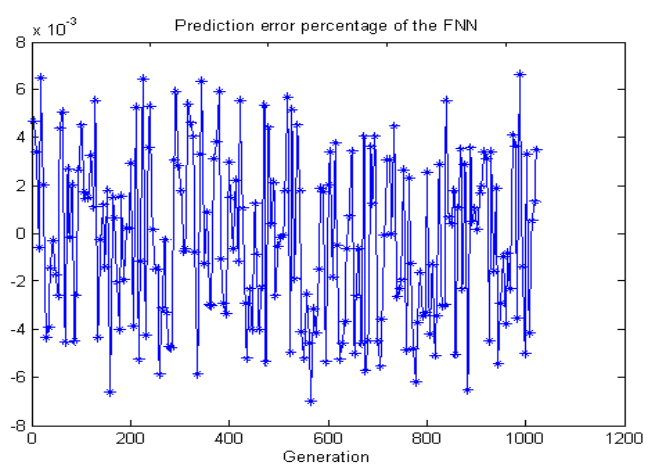

Figure 3. Prediction error percentage

\section{Application Analysis for Fuzzy Neural Network PID Controller}

In order to verify the optimization performance of combing the ACO and FNN the servo motor subsystem of the position control system is selected as the controlled object to dynamically optimize the parameters of the PID controller. Because the position control system requires the high-speed and high-precision, the servo motor need be precisely controlled. The fuzzy neural network PID controller based on the ACO algorithm is used to control the PID. The transfer function of the controlled object is shown:

$$
G(s)=\frac{20 e^{-0.5 s}}{(2 s+1)(4 s+1)(2.2 s+1)}
$$

The input signal of the transfer function is a unit step signal. The initial control parameter $K_{p}=18.00, K_{i}=5.00, K_{d}=20.00$ are selected for the conventional PID controller. The overall structure of the fuzzy neural network is set: 3-10-22-3. Learning rate $E=0.8$, inertial coefficient $\eta=0.12$, the initial range of Gaussian membership function centre $c_{i j}$ is $[-4,4]$, the initial range of the width is $(-4,4)$. The initial range of neural network weights $w_{i j}$ is $[-1,1]$. The parameters of the ACO algorithm are set: the initial ant size is 20 , the number of iteration is 1000 , the pheromone factor is 1 , the heuristic factor is 2 , the pheromone volatility coefficient is 0.05 , and the amount of pheromone is 80 . 
In the simulation, the traditional fuzzy neural network PID can find the satisfied initial weights under many randomized taking value. However, the optimized fuzzy neural network PID based on the ACO algorithm can quickly find the satisfied initial weights. The fuzzy neural network PID control is also easy to obtain the satisfied control effect. The final parameters' values of the PID controller are: $K_{p}=9.87, K_{i}=0.36, K_{d}=11.24$ 。

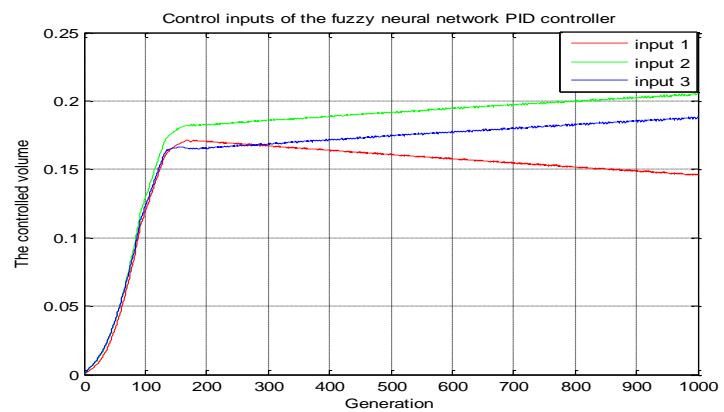

Figure 4. Control inputs of the fuzzy neural network PID controller

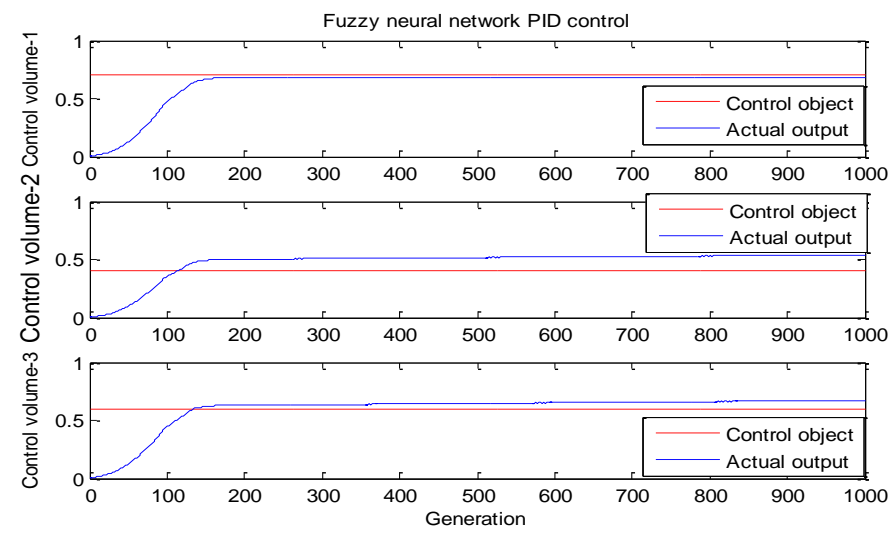

Figure 5. Fuzzy neural network PID control volume
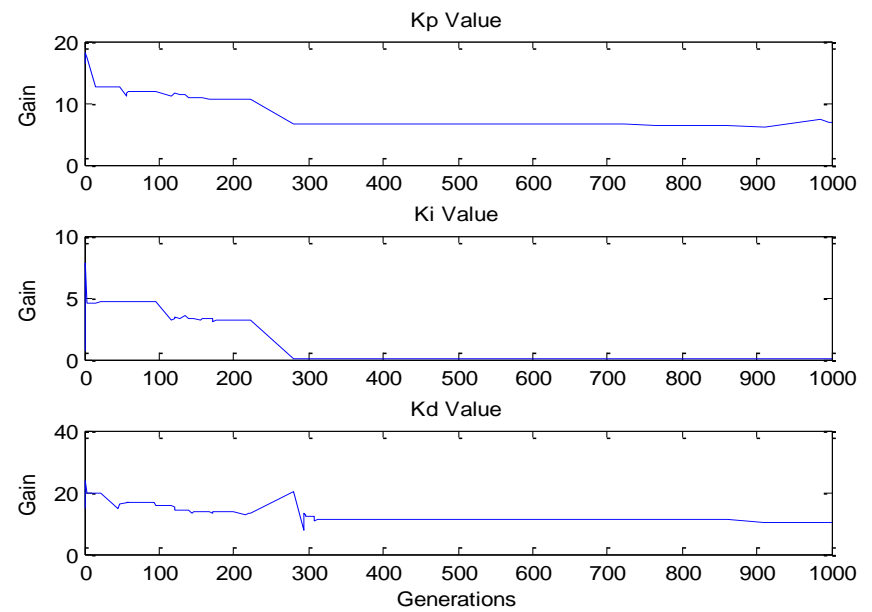

Figure 6. Parameters' values of the PID controller 


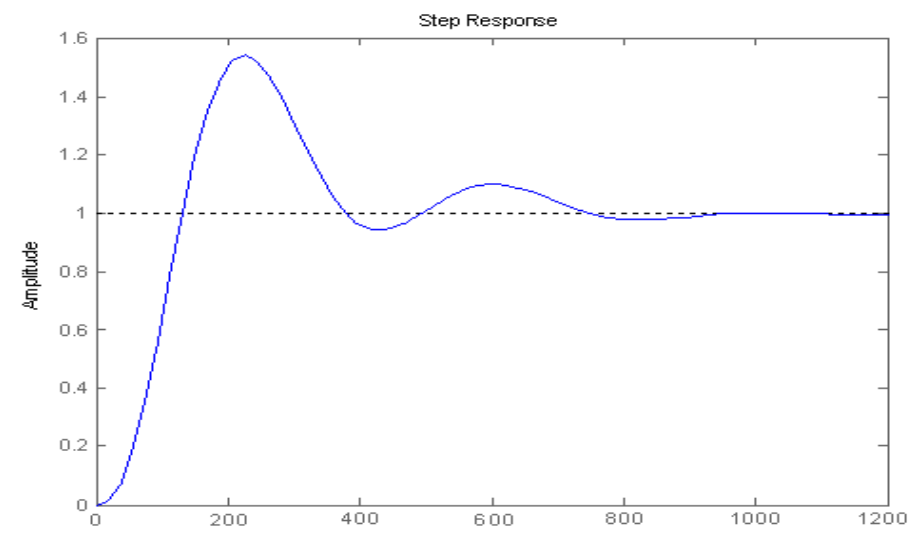

Figure 7. The process of the optimizing the PID controller

\section{Conclusions and Future Work}

The advantages of artificial intelligence techniques are used in this paper. We use the fuzzy theory, neural network and ant colony optimization algorithm to control the parameters of the PID controller. These methods do not depend on the mathematical model of the controlled object to optimize the PID controller. And the optimized PID controller takes on adaptive ability and learning ability. This optimization method may simplify the selection process of the initial weight in the fuzzy neural network PID controller and help to improve the overall performance of the control system. And it also improves the anti-interference ability of the PID controller to some extent. This method is applied in the servo motor subsystem of the position control system, the experimental results shows that this method can obtain the optimized PID controller with the fast response and small overshoot, etc. Therefore, this method has a certain potential, it is worthy of the recommended optimization design method of the fuzzy logic controller.

But in our application, we find that the effectiveness of the fuzzy neural network PID is low for solving the the parameters of the PID controller. So in next work, we will focus on research how to improve the solving effectiveness. We will try to propose a new improved method to improve the optimization effectiveness for solving the the parameters of the PID controller.

\section{ACKNOWLEDGEMENTS}

This work is partially supported by the General Scientific Research Project's Subsidize in Education Department of Liaoning Province (L2013174),the Higher growth plans of Liaoning Province for Distinguished Young Scholars(LJQ2013049),State Key Laboratory of Software Engineering (SKLSE) (SKLSE2012-09 27), the Open Project Program of Sichuan Provincial Key Lab of Process Equipment and Control (GK201202), the Open Project Program of the Traction Power State Key Laboratory of Southwest Jiaotong University (TPL1203), the Open Project Program of Guangxi Key Laboratory of Hybrid Computation and IC Design Analysis (Guangxi University for Nationalities) (2012HCIC06).The authors also gratefully acknowledge the helpful comments and suggestions of the reviewers, which have improved the presentation. 


\section{References}

[1] G. Chen, "Conventional and Fuzzy PID Cont rollers: an Overview", International Journal of Intelligent Control and Systems, vol. 1, no. 2, (1996), pp. 235-246.

[2] K. H. Ang, G. Chong and Y. Li, "PID control system analysis, design and technology", IEEE Transaction on Control System Technology, vol. 13, no. 4, (2005), pp. 559-576.

[3] S. Servet, K. Mehmet and A. Hasan, "Design and simulation of self-tuning PID-type fuzzy adaptive control for an expert HVAC system", Expert System with Application, vol. 36, no. 3, (2008), pp. 4566-4573.

[4] S. Zhang, W. T. Cyrus, J. Bentsman, A. Hussey and B. Petrus, "Simultaneous gains tuning in boiler/turbine PID-based controller clusters using iterative feedback tuning methodology", ISA Transactions, vol. 51, no. 5, (2012), pp. 609-621.

[5] D. J. Wang, "A PID controller set of guaranteeing stability and gain and phase margins for timedelay Systems", Journal of Process Control, vol. 22, no. 7, (2012), pp. 1298-1306.

[6] J. A. Romero, R. Sanchis and P. Balaguer, "PI and PID auto-tuning procedure based on simplified single parameter optimization", Journal of Process Control, vol. 21, no. 6, (2011), pp. 840-851.

[7] Z. J. Li and L. J. Wang, "Application of fuzzy control in ball mill process", Journal of North China Electric Power University, vol. 24, no. 1, (1997), pp. 1-5.

[8] Q. Zhao, "Application of fuzzy controller with neural decoupling to control system of ball mills", Engineering Journal of Wuhan University, vol. 37, no. 1, (2004), pp. 125-127.

[9] H. L. Fu and M. Li, "Variable-air-volume air-conditioning system based on PID-ANN decoupling control technology", Journal of Southwest Jiaotong University, vol. 40, no. 1, (2005), pp. 13-17.

[10] M. Dorigo and L. M. Gambardella, "Ant colony system: A cooperative learning approach to the traveling salesman problem”, IEEE Transactions on Evolutionary Computation, vol. 1, no. 1, (1997), pp. 53-66.

[11] Y. V. Pehlivanoglu and O. Baysal, "Vibrational genetic algorithm enhanced with fuzzy logic and neural networks", Aerospace Science and Technology, vol. 14, no. 1, (2010), pp. 56-64.

\section{Authors}

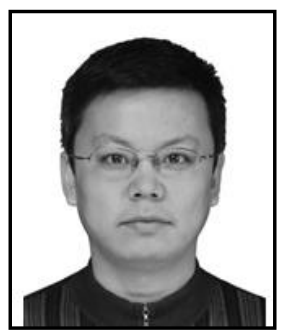

\section{Chunguang Zhang}

Lecturer, received the Engineering Master degree in traffic information engineering and control from Dalian Jiaotong University, Dalian, china, in 2005. The main research directions: industrial control network, the Internet of things, the developing of embedded system.

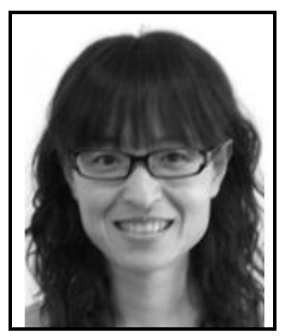

\section{Lifang Zhang}

L0ecturer, received the Engineering Master degree in traffic information engineering and control from Dalian Jiaotong University, Dalian, china, in 2006. On-job doctorate in control theory and control engineering of Dalian Maritime University, Dalian, china, since 2013. The main research directions: Fieldbus Control System network, industrial process control. 